\title{
Urine Cotinine Should Be Involved in Initial Evaluation of Tinnitus in Adolescents
}

\author{
Doh Young Lee · Young Ho Kim \\ Department of Otorhinolaryngology-Head and Neck Surgery, SMG-SNU Boramae Medical Center, \\ Seoul National University College of Medicine, Seoul, Korea
}

Objectives. Smoking is associated with hearing loss, while the correlation between tinnitus and smoking is not fully elucidated. This study aimed to evaluate risk factors of tinnitus in adolescents in terms of smoking, and we identified a rectifiable parameter that can be serially monitored.

Methods. A cross-sectional study was conducted using data from the Korea National Health and Nutrition Examination Survey, with 2,782 participants aged 12 to 18 years, from 2008 through 2011. Participants with history of ear disease, hearing loss, and inadequate responses to questionnaires were excluded. We investigated the prevalence of tinnitus and tinnitus-related annoyance by questionnaire and sought potential risk factors in blood and urine tests and smoking history.

Results. The prevalence of tinnitus in the 12- to 18 -year-old population was $17.5 \%$, with $3.3 \%$ reporting tinnitus-related annoyance. On univariate analysis, the prevalence of tinnitus increased with age $(P<0.001)$ and was higher among girls $(P=0.012)$. Blood tests and urinalysis showed significant correlation between tinnitus and red blood cell count, alkaline phosphatase levels, and urine cotinine $(P=0.002, P<0.001, P=0.018$, respectively). In multivariate analysis, the urine cotinine level was the only parameter associated with tinnitus (odds ratio, 1.000; 95\% confidence interval, 0.999 to $1.000 ; P=0.038)$. Smoking was also significantly correlated with tinnitus $(P=0.043)$, and amount of smoking with tinnitus-related annoyance $(P=0.045)$. However, current smoking and past smoking were not correlated with tinnitus.

Conclusion. Urine cotinine may be a rectifiable marker for management of tinnitus in adolescents. This suggests that smoking cessation should be incorporated in the management of tinnitus in adolescents.

Keywords. Tinnitus; Annoyance; Smoking; Cotinine

\section{INTRODUCTION}

Although tinnitus is thought to be an uncommon symptom in the pediatric population, its incidence is known to be not low [1].The reported prevalence of tinnitus in childhood varies from $7 \%$ to $34 \%$ among individuals with no hearing loss and can

\footnotetext{
- Received November 23, 2017

Revised January 22, 2018

Accepted February 4, 2018

- Corresponding author: Young Ho Kim

Department of Otorhinolaryngology-Head and Neck Surgery, SMG-SNU Boramae Medical Center, Seoul National University College of Medicine, 20 Boramae-ro 5-gil, Dongjak-gu, Seoul 07061, Korea

Tel: +82-2-870-2442, Fax: +82-2-870-3863

E-mail: yhkiment@gmail.com
}

reach $66 \%$ among those with moderate to severe hearing loss $[2,3]$. Several large cohort studies have recently revealed that $7 \%$ to $17 \%$ of adolescents aged 12 to 19 report tinnitus [4,5], which is similar to the prevalence of tinnitus in adults $[6,7]$. However, tinnitus in children tends to be neglected because people in this age group rarely mention the symptom spontaneously to their parents, and they report tinnitus infrequently when visiting ear clinics [8].

Several risk factors have been associated with pediatric tinnitus. Hearing loss and noise exposure are known to be risk factors for tinnitus in the youth population [9]. Female sex, low socioeconomic status of the family, and history of ear infection have also been associated with tinnitus in adolescents [10-12]. To date, there has been a lack of studies evaluating the correla-

Copyright (C) 2018 by Korean Society of Otorhinolaryngology-Head and Neck Surgery.

This is an open-access article distributed under the terms of the Creative Commons Attribution Non-Commercial License (http://creativecommons.org/licenses/by-nc/4.0)

which permits unrestricted non-commercial use, distribution, and reproduction in any medium, provided the original work is properly cited. 
tion of serologic/urinary parameters and nutrition status with tinnitus, although some studies have reported an association between smoking and tinnitus [13]. Considering that medical treatments are burdensome, and because there is a lack of evidence-based information regarding titration and duration of medical treatment for pediatric tinnitus, evaluation of rectifiable factors such as nutrition status and social habits would be far more useful. Although routine examination of blood and urine for contributing factors is frequently performed, significant markers are not frequently observed in pediatric populations.

Smoking among adolescents and young adults has decreased rapidly and unprecedentedly. However, there are still a considerable number of cigarette smokers in this population $[14,15]$. Moreover, there is a possible association between direct or indirect smoking, various diseases, and malnutrition in adolescents [16]. A comprehensive search for an association between tinnitus and smoking status may be important in pediatric tinnitus. In this study, we aimed to evaluate risk factors for tinnitus in adolescents in terms of smoking status, and we identified a novel marker for serial follow-up and monitoring.

\section{MATERIALS AND METHODS}

\section{Data collection}

This study analyzed data from the fourth and fifth Korea National Health and Nutrition Examination Survey (KNHANES) from 2008 to 2011. The KNHANES is an ongoing nationwide survey of noninstitutionalized individuals in South Korea with a sophisticated level of standardization and quality control. The data were collected and managed by the Centers for Disease Control and Prevention of Korea. Each year, a panel selected 192 enumeration districts and 20 households in each district for proper sampling to reflect the entire Korean population. The sampling was weighted by statisticians by adjusting the poststratification, nonresponse rate and extreme values. These data represent the civilian, noninstitutionalized South Korean population using stratified, multistage clustered sampling based on national census data from the National Statistical Office.

\section{H I G}

- This cross-sectional study involved 2,782 participants from the Korea National Health and Nutrition Examination Survey data.

- Urine cotinine was an independent factor associated with tinnitus.

- Amount of smoking was also significantly associated with tinnitus-related annoyance.

- However, the history of current/past smoking was not correlated with tinnitus.
37,753 Total of participants

$(9,744$ in $2008,10,533$ in $2009,8,958$ in 2010 , and 8,518 in 2011)

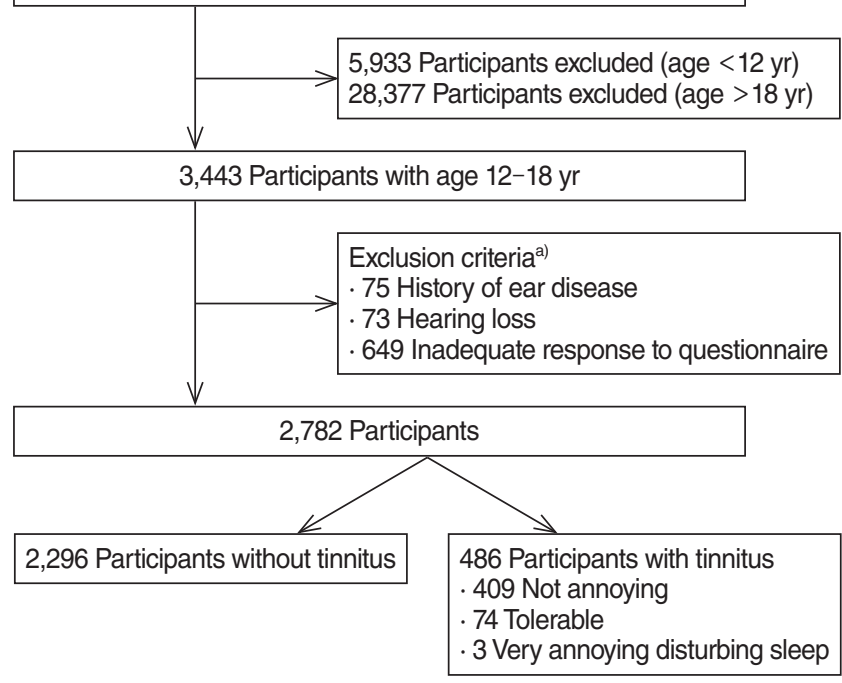

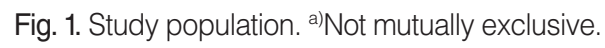

\section{Survey and examination}

Among a total of 37,753 participants (9,744 in 2008, 10,533 in $2009,8,958$ in 2010 , and 8,518 in 2011), there were 3,443 adolescents, aged 12 to 18 years, enrolled in this study (Fig. 1). Further validation of significant parameters in adolescents was performed in 19,384 adult participants aged $>19$ years. Written informed consent was obtained from all participants prior to the survey. All tympanic membranes (right and left) were examined for signs of prior ear disease by trained otologists and were categorized into three groups: normal, abnormal, and could not examine. Pure-tone audiometry for thresholds was measured at $500,1,000,2,000,3,000,4,000$, and $6,000 \mathrm{~Hz}$ in both ears in a soundproof booth with an automatic audiometer. Hearing loss was defined as more than an average of $40 \mathrm{~dB}$ hearing loss on pure-tone audiometry at 500,1,000, 2,000, and 3,000 Hz.

The presence of tinnitus was defined according to the response (yes/no/cannot remember) to the question, "Have you heard any ringing, buzzing, roaring, or hissing sounds without an external acoustic source in the past year?" Participants were then asked about the severity of the tinnitus, i.e., "Do these sounds bother you?" The response options to the second question were "No," "A little annoying," and "Very annoying and disrupting sleep."

Age, sex, body mass index (BMI), and smoking history were categorized as personal risk factors in this study. Current and past smoking history was surveyed, and daily amount smoked was surveyed for smokers. Smoking habits of parents were also surveyed. Blood was examined for red blood cell count, hemoglobin levels, hematocrit, white blood cell count, serum blood urea nitrogen, creatinine, glucose, aspartate aminotransferase (glutamic oxaloacetic transaminase), alanine aminotransferase 
(glutamate pyruvate transaminase), triglyceride, total cholesterol, high-density lipoprotein, alkaline phosphatase (ALP), lead $(\mathrm{Pb})$, mercury $(\mathrm{Hg})$, and cadmium $(\mathrm{Cd})$ levels and urine was examined for $\mathrm{pH}$ and specific gravity and for protein, glucose, ketone, blood, bilinogen, cotinine, and creatinine levels.

\section{Statistical analysis}

A sample weight for each participant, as reported by the KNHANES for every individual based on the complex probability of being selected, was applied in all statistical analyses. After applying the weighted values recommended by KNHANES, the adjusted odds ratios (AOR) were estimated. Associations between various parameters and tinnitus were analyzed using multiple logistic regression analysis. To evaluate the collinearity, multiple linear regression analysis was performed and variance inflation factors are assessed. In subgroup analysis, the difference in risk factors between individuals with and without tinnitus was analyzed by Student $t$-test. AOR and $95 \%$ confidence intervals $(\mathrm{CI})$ were calculated, and $P$-values $<0.05$ were considered to indicate significance. The statistical analyses were performed with IBM SPSS ver. 20.0 (IBM Corp., Armonk, NY, USA).

\section{RESULTS}

\section{Study population}

Among 3,443 enrollees, individuals meeting the following criteria were excluded: (1) history of ear disease, as reported by the respondents or observed by examination of the tympanic membranes ( $n=75)$; (2) detection of unilateral or bilateral hearing loss by the hearing test $(n=73)$; and (3) inadequate response to questionnaire regarding tinnitus, tinnitus-related annoyance, and smoking history ( $n=649$ ) (Fig. 1 ). Finally, a total of 2,782 individuals with a mean age of 14.8 years (range, 12 to 18 years) and a male to female ratio of $1.13: 1$ were included. The prevalence of tinnitus in the total cohort was $17.5 \%(n=486)$. Among the participants with tinnitus, $84.2 \%(n=409)$ reported no annoyance from tinnitus, $15.2 \%(n=74)$ reported tolerable annoyance, and $0.6 \%(n=3)$ reported severe annoyance. All 77 individuals who reported any degree of annoyance were combined into a single category, tinnitus-related annoyance, for further analysis. There was a significant $(P<0.001)$ increase in the prevalence of tinnitus with age, and the prevalence of smoking also increased significantly $(P<0.001)$ with age (Fig. 2$)$. The prevalence of tinnitus-related annoyance did not differ significantly among age groups $(P=0.404)$.

\section{Risk factors for tinnitus}

On univariate analysis, age, sex, and BMI were significantly associated with tinnitus $(P<0.001, P=0.012$, and $P=0.045$, respectively) (Table 1$)$, with a greater prevalence among girls and

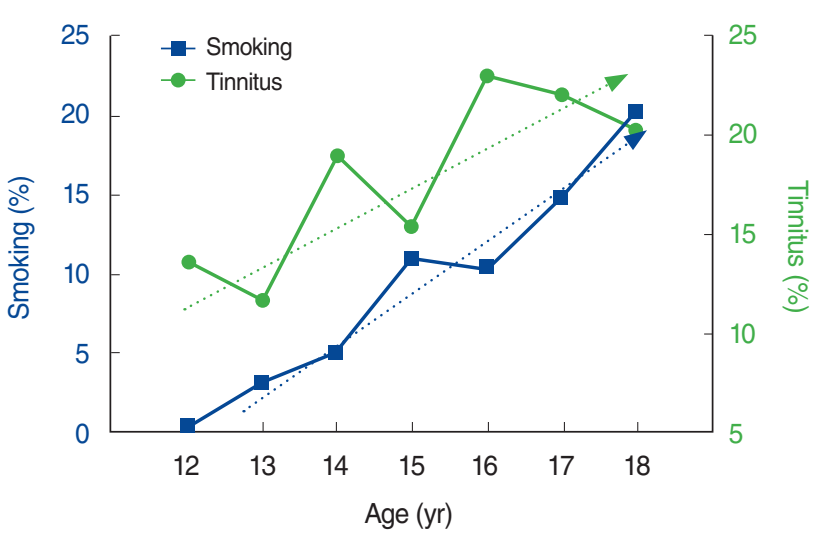

Fig. 2. Prevalence of smoking and tinnitus in each age group. The prevalence of tinnitus increased with age $(P<0.001)$, as did the proportion of smokers $(P<0.001)$, albeit with some fluctuation.

among individuals with higher BMI. Current smoking was also significantly correlated with tinnitus $(P=0.043)$, but there was no difference in the amount smoked between individuals with and without tinnitus $(P=0.677)$. Regarding laboratory values, the red blood cell count, serum ALP level, and urine cotinine level were significantly associated with tinnitus $(P=0.002$, $P<0.001$, and $P=0.018$, respectively) on univariate analysis. ALP levels were lower and urine cotinine levels were higher among individuals with tinnitus compared to those without tinnitus. However, the urine cotinine level was the only parameter associated with tinnitus on multivariate analysis (OR, 1.000; $95 \% \mathrm{CI}, 0.999$ to $1.000 ; P=0.038$ ) (Table 1$)$. When the overall cohort was categorized according to smoking history and sex, the urine cotinine level was consistently higher in individuals with tinnitus in all subgroups (Fig. 3A, B), reaching statistical significance with smoking and male sex $(P=0.036$ and $P=0.021$, respectively). There was no blood or urine parameter showing significant correlation with tinnitus among nonsmokers and girls. Urine cotinine was also significantly correlated with tinnitus in the total population of adult participants $(P=0.008)$, while subgroup analyses according to smoking history and sex did not reach statistical significance (Supplementary Fig. 1).

\section{Factors associated with tinnitus-related annoyance}

The amount smoked was the only factor that was significantly correlated with tinnitus-related annoyance (Fig. 3C). Table 2 shows the correlation analysis between tinnitus-related annoyance and the parameters that were significantly correlated with tinnitus (age, sex, BMI, smoking history, serum ALP level, and urine cotinine level), none of which reached statistical significance $(P=0.437, P=0.240, P=0.502, P=0.962, P=0.132$, and $P=0.141$, respectively) for tinnitus-related annoyance. In adult participants, tinnitus-related annoyance was not correlated with urine cotinine $(P=0.472)$, but was significantly associated with the amount smoked $(P=0.020)$ (Supplementary Fig. 2). 
Table 1. Univariate and multivariate binary logistic regression analysis of risk factors for tinnitus

\begin{tabular}{|c|c|c|c|c|c|c|c|c|c|}
\hline \multirow{2}{*}{ Variable } & \multicolumn{5}{|c|}{ Univariate analysis } & \multicolumn{4}{|c|}{ Multivariate analysis } \\
\hline & Tinnitus (+) & Tinnitus (-) & OR & $95 \% \mathrm{Cl}$ & $P$-value & OR & $95 \% \mathrm{Cl}$ & $P$-value & VIF \\
\hline Age (yr) & $15.1 \pm 1.9$ & $14.7 \pm 2.0$ & 0.893 & $0.850-0.938$ & $<0.001$ & 0.958 & $0.860-1.066$ & 0.428 & 2.222 \\
\hline Sex (male:female) & $1: 1.09$ & $1: 0.84$ & 1.286 & $1.057-1.565$ & 0.012 & 1.309 & $0.864-1.983$ & 0.203 & 2.212 \\
\hline Body mass index $\left(\mathrm{kg} / \mathrm{m}^{2}\right)$ & $21.15 \pm 3.66$ & $20.80 \pm 3.55$ & 0.973 & $0.947-0.999$ & 0.045 & 0.968 & $0.930-1.007$ & 0.103 & 1.095 \\
\hline Current smoking history & $49(10.1)$ & $183(8.0)$ & 1.286 & $1.014-1.809$ & 0.043 & 1.275 & $0.663-2.450$ & 0.467 & 1.197 \\
\hline Parental smoking history & $133(27.4)$ & $583(25.4)$ & 0.598 & $0.071-2.030$ & 0.636 & - & - & - & - \\
\hline \multicolumn{10}{|l|}{ Blood test } \\
\hline Red blood cell $\left(\times 10^{6} / \mu \mathrm{L}\right)$ & $4.79 \pm 0.41$ & $4.85 \pm 0.40$ & 1.503 & $1.160-1.947$ & 0.002 & 1.193 & $0.758-1.878$ & 0.445 & 1.698 \\
\hline Hemoglobin (g/dL) & $13.95 \pm 1.32$ & $13.06 \pm 1.28$ & 1.072 & $0.990-1.160$ & 0.087 & - & - & - & - \\
\hline Hematocrit (\%) & $41.60 \pm 3.42$ & $41.88 \pm 3.31$ & 1.026 & $0.995-1.058$ & 0.106 & - & - & - & - \\
\hline White blood cell $\left(\times 10^{9} / L\right)$ & $6.15 \pm 1.43$ & $6.15 \pm 1.42$ & 0.997 & $0.928-1.071$ & 0.934 & - & - & - & - \\
\hline Blood urea nitrogen (mg/dL) & $11.77 \pm 2.66$ & $11.73 \pm 2.88$ & 0.995 & $0.960-1.031$ & 0.771 & - & - & - & - \\
\hline Creatinine (mg/dL) & $0.74 \pm 0.14$ & $0.73 \pm 0.14$ & 0.812 & $0.400-1.647$ & 0.564 & - & - & - & - \\
\hline Glucose (mg/dL) & $88.28 \pm 6.31$ & $88.95 \pm 8.35$ & 1.014 & $0.998-1.030$ & 0.088 & - & - & - & - \\
\hline Glutamic oxaloacetic transaminase (IU/L) & $17.89 \pm 6.02$ & $18.41 \pm 7.83$ & 1.013 & $0.994-1.031$ & 0.176 & - & - & - & - \\
\hline Glutamate pyruvate transaminase (IU/L) & $14.89 \pm 14.37$ & $14.48 \pm 13.27$ & 0.998 & $0.991-1.005$ & 0.557 & - & - & - & - \\
\hline Triglyceride (mg/dL) & $83.67 \pm 42.55$ & $85.05 \pm 50.94$ & 1.001 & $0.998-1.003$ & 0.592 & - & - & - & - \\
\hline Total cholesterol (mg/dL) & $156.01 \pm 26.77$ & $156.07 \pm 27.40$ & 1.000 & $0.996-1.004$ & 0.966 & - & - & - & - \\
\hline High-density lipoprotein (mg/dL) & $49.20 \pm 9.04$ & $49.47 \pm 9.47$ & 1.003 & $0.992-1.014$ & 0.579 & - & - & - & - \\
\hline Alkaline phosphatase (IU/L) & $460.45 \pm 293.33$ & $529.94 \pm 321.63$ & 1.001 & $1.000-1.001$ & $<0.001$ & 1.000 & $0.999-1.001$ & 0.779 & 2.713 \\
\hline $\mathrm{Pb}(\mu \mathrm{g} / \mathrm{dL})$ & $1.35 \pm 0.53$ & $0.35 \pm 0.48$ & 1.015 & $0.674-1.529$ & 0.942 & - & - & - & - \\
\hline $\mathrm{Hg}(\mathrm{ng} / \mathrm{mL})$ & $2.46 \pm 1.24$ & $2.26 \pm 1.22$ & 0.887 & $0.762-1.033$ & 0.122 & - & - & - & - \\
\hline $\mathrm{Cd}(\mathrm{ng} / \mathrm{mL})$ & $0.41 \pm 0.31$ & $0.41 \pm 0.25$ & 0.816 & $0.393-1.691$ & 0.584 & - & - & - & - \\
\hline \multicolumn{10}{|l|}{ Urine test } \\
\hline $\mathrm{pH}$ & $5.62 \pm 0.70$ & $5.60 \pm 0.71$ & 0.950 & $0.819-1.103$ & 0.501 & - & - & - & - \\
\hline Specific gravity & $1.02 \pm 0.01$ & $1.02 \pm 0.01$ & 0.304 & $0.000-1.611$ & 0.893 & - & - & - & - \\
\hline Protein (mg/dL) & $0.15 \pm 0.41$ & $0.15 \pm 0.41$ & 1.042 & 0.799-1.359 & 0.762 & - & - & - & - \\
\hline Ketone (mg/dL) & $0.24 \pm 0.58$ & $0.23 \pm 0.53$ & 0.957 & $0.788-1.162$ & 0.659 & - & - & - & - \\
\hline Bilirubin (mg/dL) & $0.40 \pm 0.80$ & $0.38 \pm 0.78$ & 0.972 & $0.850-1.111$ & 0.673 & - & - & - & - \\
\hline Blood & $0.40 \pm 0.87$ & $0.40 \pm 0.84$ & 1.006 & $0.886-1.142$ & 0.928 & - & - & - & - \\
\hline Bilinogen (mg/dL) & $0.06 \pm 0.25$ & $0.05 \pm 0.23$ & 0.853 & 0.559-1.302 & 0.462 & - & - & - & - \\
\hline Cotinine (ng/mL) & $128.76 \pm 388.70$ & $77.56 \pm 276.89$ & 1.000 & $0.999-1.000$ & 0.018 & 0.999 & $0.999-1.000$ & 0.038 & 1.185 \\
\hline Creatinine $(\mathrm{mg})$ & $205.35 \pm 92.24$ & $201.51 \pm 89.75$ & 1.000 & $0.998-1.000$ & 0.575 & - & - & - & - \\
\hline
\end{tabular}

Values are presented as mean \pm standard deviation or number (\%).

$\mathrm{OR}$, odds ratio; $\mathrm{Cl}$, confidence interval; VIF, variance inflation factor.

\section{DISCUSSION}

This study demonstrated that considerable proportion of adolescents, $17.5 \%$, experienced tinnitus. In the total cohort, $3 \%$ reported tinnitus-related annoyance, while $15.8 \%$ of those specifically reporting tinnitus also reported tinnitus-related annoyance. Increased age, female sex, and smoking were risk factors for tinnitus on univariate analysis. The amount smoked did not correlate with the prevalence of tinnitus, but there was a significant association between amount smoked and tinnitus-related annoyance. Urine cotinine level was the only parameter associated with tinnitus in multivariate analysis. In the subgroup analysis, urine cotinine was significantly correlated with tinnitus in smokers and in boys.

The role of smoking in hearing loss remains controversial although there is agreement regarding the adverse effects of smoking on the middle ear [17,18]. Fransen et al. [19] and Cruickshanks et al. [20] have reported an association between hearing and smoking, but no such association was found in the Framingham cohort. Nonetheless, smoking is consistently identified as a risk factor for tinnitus in both adults and children [21,22], and several large cohort studies have recently confirmed that direct and indirect smoking is a significant risk factor in pediatric tinnitus $[4,5,9]$. Our finding of a significant correlation between current smoking and tinnitus corroborates these results. Hearing loss is not the only pathophysiologic entity in tinnitus [23-25], and it is possible that smoking affects the development of tinnitus by a different mechanism than hearing loss.

Although the exact pathophysiology of tinnitus is beyond the scope of this study, we propose that there is a possibility that smoking and tinnitus are directly related. Smoking can influence all of the vital structures of the auditory tract. Cochlear blood 


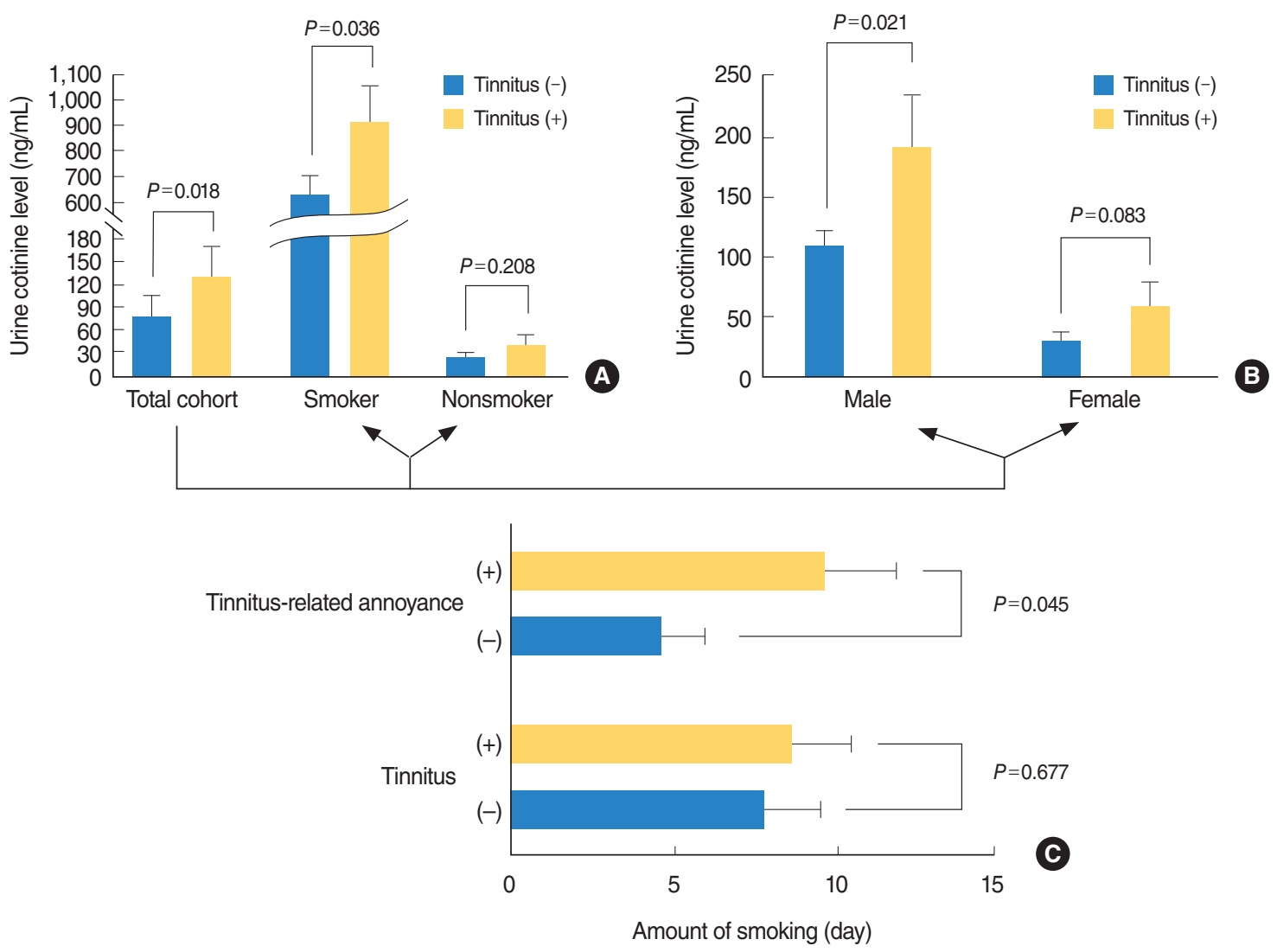

Fig. 3. Variation in urine cotinine levels by smoking history $(A)$ and sex $(B)$, and correlation between amount smoked, tinnitus, and tinnitus-related annoyance in smokers (C). (A, B) Participants with tinnitus showed higher urine cotinine levels than those without tinnitus in all subgroups, which reached statistical significance in the total cohort and in smokers and boys. (C) Although development of tinnitus was not associated with the amount smoked, tinnitus-related annoyance was significantly correlated with amount smoked.

Table 2. Risk factors for tinnitus-related annoyance

\begin{tabular}{|c|c|c|c|c|c|}
\hline Variable & Tinnitus-related annoyance $(+)$ & Tinnitus-related annoyance (-) & OR & $95 \% \mathrm{Cl}$ & $P$-value \\
\hline Age (yr) & $15.3 \pm 2.1$ & $15.1 \pm 1.9$ & 1.051 & $0.927-1.193$ & 0.437 \\
\hline Sex (male:female) & $1: 1.41$ & $1: 1.05$ & 0.744 & $0.454-1.219$ & 0.240 \\
\hline Body mass index $\left(\mathrm{kg} / \mathrm{m}^{2}\right)$ & $20.9 \pm 3.5$ & $21.2 \pm 3.7$ & 0.977 & $0.912-1.046$ & 0.502 \\
\hline Current smoking history & $8(10.4)$ & $40(9.8)$ & 1.021 & $0.436-2.392$ & 0.962 \\
\hline Alkaline phosphatase (IU/L) & $414.2 \pm 241.2$ & $471.7 \pm 304.1$ & 0.999 & $0.998-1.000$ & 0.132 \\
\hline Cotinine (ng/mL) & $211.9 \pm 606.4$ & $107.8 \pm 327.7$ & 1.001 & $1.000-1.001$ & 0.141 \\
\hline
\end{tabular}

Values are presented as mean \pm standard deviation or number (\%).

OR, odds ratio; $\mathrm{Cl}$, confidence interval.

supply may be decreased with smoking because of smoking-related increases in blood viscosity and reduced available oxygen [26]. Negley et al. [27] and Torre et al. [28] evaluated young adult smokers with normal hearing and found that otoacoustic emissions were significantly reduced in smokers. Nicotine and smoke-related toxins may also influence the auditory cortex by reducing metabolic and transcription activities [29]. In addition, there is a considerable amount of evidence pointing to smoking as a risk factor in adult tinnitus [6,13,21], and this was supported by our results. Considering that adolescents, who are in a growing period, may be more susceptible to impacts from smok- ing, impairment of the normal central auditory transduction system by smoking could be more severe in adolescents than in adults [30].

In our study, we divided total cohort into subgroups for following reasons. With regard to the subgroups of smokers and nonsmokers, we aimed to show the level of cotinine which is much higher and can be the reference for separating smoker from nonsmokers by cotinine level without history taking. In terms of the subgroups according to the sex, we aimed show that cotinine level can be more powerful marker in boys. As discussed later, etiology of tinnitus in girls can be more complicated 
because they are more susceptible to various factors. They may have impact on quality of life of various adverse effects of tinnitus, including pain, sleep difficulties, and lack of energy, all of which can also influence their perception of tinnitus.

Our novel finding is that urine cotinine levels are significantly correlated with tinnitus. There has been no prior study that has evaluated this biomarker for pediatric tinnitus. Nicotine is a specific biomarker of exposure to tobacco smoke, but it is limited in this task because of its short half-life ( 1 to 3 hours) [31]. Cotinine, which is the primary proximate metabolite of nicotine, is used more frequently as a biomarker of tobacco smoke exposure because its half-life approximately 16 to 18 hours, and its levels remain fairly constant throughout the day [32]. Several studies have shown that the urine cotinine level is well correlated with current smoking status even among individuals with chronic disease [33,34]. In our multivariate analysis, the urine cotinine level was the only factor associated with tinnitus in adolescents, and on subgroup analysis, it was significantly associated with tinnitus in smokers and in boys. Because we did not identify any specific risk factors for tinnitus in nonsmokers or girls, further analysis should be performed in these subgroups to elucidate the risk factors for tinnitus. There was also a significant association between the urine cotinine level and tinnitus among the adult participants in our study, and the lack of statistical significance of any risk factor in our adult subgroups (smokers, nonsmokers, men, and women) may have been a function of confounding factors such as chronic disease and medication use. Conversely, the urine cotinine level could be a more powerful indicator for tinnitus in adolescents because there should be relatively fewer confounding factors than would be expected in adults.

Furthermore, the smoking behaviors of caregivers can affect urine cotinine levels in children [35]. While there is not yet consensus regarding an association between tinnitus and passive smoking in the pediatric population, a higher prevalence of tinnitus has been observed among passive smokers [4]. We did not identify a correlation between parental smoking history and tinnitus in children although the participants with tinnitus did show higher urine cotinine levels than those without tinnitus (not statistically significant). Considering that there is a discrepancy between smoking history and urine cotinine level, detailed history taking is very important with regard to the source of exposure to smoking. This study was based on the survey, and recalling error and intentional false response can be the reason for the discrepancy. Moreover, indirect exposure from care-givers, friends, or other places where there are a lot of smokers can be the reason for elevated urine cotinine which is not in accordance with smoking history. Additional clinical studies regarding the correlation between tinnitus and passive smoking, with more refined measurements of indirect smoking, are needed for clarification. Meanwhile, efforts to identify major sources of smoke exposure and the means of limiting passive smoking may be needed for nonsmokers.

Cotinine is now widely used as a biomarker for various smoking-associated health problems, including cardiovascular disease [36], and here, we postulate the importance of urine cotinine as a biomarker of tinnitus in adolescents. According to our results, we recommend that in addition to any basic audiologic, serologic, and radiologic examination, physicians should check urine cotinine levels in adolescent patients with tinnitus and should obtain smoking histories from both the patient and the patient's parents. Urine cotinine should be included in the initial evaluation because cotinine can reflect the smoking status and can therefore reflect a potential risk factor for treating tinnitus. Monitoring of urine cotinine levels serve as a useful measure of smoke exposure in active and passive smokers, while in nonsmokers, elevated urine cotinine levels might signal unidentified sources of smoke exposure that can be uncovered by a more comprehensive history-taking.

In the present study, there were associated factors other than smoking, including age, sex, alcohol consumption, and ALP. As shown in Fig. 1, the proportion of the smoking population increased with age. In addition, ALP in younger adolescents is usually higher than older members of that age group $[37,38]$. Participants in this study showed a trend of significantly decreasing of ALP with increasing age (Supplementary Fig. 3). Considering that smoking was the most influential factor, we thought age and ALP might have been confounders with their association with smoking.

The response to noxious stimuli may vary according to sex [39]. Typically, female respondents have reported greater impact on quality of life of various adverse effects of tinnitus, including pain, sleep difficulties, and lack of energy, all of which can also influence their perception of tinnitus [40,41]. Some authors have reported that tinnitus in childhood and adolescence is more common in girls $[5,10]$, and our univariate analysis in the adolescents supported this, although the association did not persist through the multivariate analysis. Further study among girls with tinnitus might further clarify both modifiable and nonmodifiable risk factors.

Although we analyzed a variety of potential risk factors, our study was limited by its cross-sectional nature, and we could not rule out the possibility of reverse causality. In addition, a considerable amount of data in this study relied on subjective recall of the participants, thus creating a potential recall bias. Another limitation is that risk factors including comorbidities and medications that can cause or aggravate tinnitus were omitted.

In conclusion, we found that that urine cotinine level is a significant marker for tinnitus in adolescents. Because smoking is a known risk factor for tinnitus, adolescents with tinnitus should be questioned regarding their smoking habits and supportive measures toward smoking cessation, with serial monitoring of urine cotinine levels, should be incorporated in the treatment of tinnitus. For nonsmokers, elevated urine cotinine should prompt 
a closer search for sources of passive smoke that can be eliminated.

\section{CONFLICT OF INTEREST}

No potential conflict of interest relevant to this article was reported.

\section{ACKNOWLEDGMENTS}

This study was supported by a clinical research grant provided from SMG-SNU Boramae Medical Center, Seoul, Republic of Korea.

We thank the 150 residents of the otorhinolaryngology departments of 47 training hospitals in South Korea and members of the Division of Chronic Disease Surveillance in Korea Centers for Disease Control and Prevention for participating in this survey and the dedicated work they provided.

\section{SUPPLEMENTARY MATERIALS}

Supplementary materials can be found via https://doi.org/10. 21053/ceo.2017.01641.

\section{REFERENCES}

1. Savastano M, Marioni G, de Filippis C. Tinnitus in children without hearing impairment. Int J Pediatr Otorhinolaryngol. 2009 Dec;73 Suppl 1:S13-5.

2.Aust G.Tinnitus in childhood. IntTinnitus J. 2002;8(1):20-6.

3. Savastano M. Characteristics of tinnitus in childhood. Eur J Pediatr. 2007 Aug;166(8):797-801.

4. Mahboubi H, Oliaei S, Kiumehr S, Dwabe S, Djalilian HR. The prevalence and characteristics of tinnitus in the youth population of the United States. Laryngoscope. 2013 Aug;123(8):2001-8.

5. Park B, Choi HG, Lee HJ, An SY, Kim SW, Lee JS, et al. Analysis of the prevalence of and risk factors for tinnitus in a young population. Otol Neurotol. 2014 Aug;35(7):1218-22.

6. Kim HJ, Lee HJ, An SY, Sim S, Park B, Kim SW, et al. Analysis of the prevalence and associated risk factors of tinnitus in adults. PLoS One. 2015 May;10(5):e0127578.

7. Gallus S, Lugo A, Garavello W, Bosetti C, Santoro E, Colombo P, et al. Prevalence and determinants of tinnitus in the Italian adult population. Neuroepidemiology. 2015 Aug;45(1):12-9.

8. Kim YH, Jung HJ, Kang SI, Park KT, Choi JS, Oh SH, et al. Tinnitus in children: association with stress and trait anxiety. Laryngoscope. 2012 Oct;122(10):2279-84

9. Coelho CB, Sanchez TG, Tyler RS. Tinnitus in children and associated risk factors. Prog Brain Res. 2007 Oct;166:179-91.

10. Henderson E, Testa MA, Hartnick C. Prevalence of noise-induced hearing-threshold shifts and hearing loss among US youths. Pediatrics. 2011 Jan;127(1):e39-46.

11. Niskar AS, Kieszak SM, Holmes AE, Esteban E, Rubin C, Brody DJ.
Estimated prevalence of noise-induced hearing threshold shifts among children 6 to 19 years of age: the Third National Health and Nutrition Examination Survey, 1988-1994, United States. Pediatrics. 2001 Jul;108(1):40-3.

12. Bulbul SF, Muluk NB, Cakir EP, Tufan E. Subjective tinnitus and hearing problems in adolescents. Int J Pediatr Otorhinolaryngol. 2009 Aug;73(8):1124-31.

13. Brunnberg E, Linden-Bostrom M, Berglund M.Tinnitus and hearing loss in 15-16-year-old students: mental health symptoms, substance use, and exposure in school. Int J Audiol. 2008 Nov;47(11):688-94.

14. Lantz PM. Smoking on the rise among young adults: implications for research and policy.Tob Control. 2003 Jun;12 Suppl 1:i60-70.

15. Nelson DE, Mowery P, Asman K, Pederson LL, O’Malley PM, Malarcher A, et al. Long-term trends in adolescent and young adult smoking in the United States: metapatterns and implications. Am J Public Health. 2008 May;98(5):905-15.

16. Best CM, Sun K, de Pee S, Bloem MW, Stallkamp G, Semba RD. Parental tobacco use is associated with increased risk of child malnutrition in Bangladesh. Nutrition. 2007 Oct;23(10):731-8.

17. Sharabi Y, Reshef-Haran I, Burstein M, Eldad A. Cigarette smoking and hearing loss: lessons from the young adult periodic examinations in Israel (YAPEIS) database. Isr Med Assoc J. 2002 Dec;4(12): 1118-20.

18. Agius AM, Wake M, Pahor AL, Smallman LA. Smoking and middle ear ciliary beat frequency in otitis media with effusion. Acta Otolaryngol. 1995 Jan;115(1):44-9.

19. Fransen E, Topsakal V, Hendrickx JJ, Van Laer L, Huyghe JR, Van Eyken E, et al. Occupational noise, smoking, and a high body mass index are risk factors for age-related hearing impairment and moderate alcohol consumption is protective: a European populationbased multicenter study. J Assoc Res Otolaryngol. 2008 Sep;9(3): 264-76.

20. Cruickshanks KJ, Klein R, Klein BE, Wiley TL, Nondahl DM, Tweed TS. Cigarette smoking and hearing loss: the epidemiology of hearing loss study. JAMA. 1998 Jun;279(21):1715-9.

21. Martines F, Sireci F, Cannizzaro E, Costanzo R, Martines E, Mucia $\mathrm{M}$, et al. Clinical observations and risk factors for tinnitus in a Sicilian cohort. Eur Arch Otorhinolaryngol. 2015 Oct;272(10):2719-29.

22. Chang J, Ryou N, Jun HJ, Hwang SY, Song JJ, Chae SW. Effect of cigarette smoking and passive smoking on hearing impairment: data from a population-based study. PLoS One. 2016 Jan;11(1):e0146608.

23. Langguth B. Treatment of tinnitus. Curr Opin Otolaryngol Head Neck Surg. 2015 Oct;23(5):361-8.

24. Yang S, Weiner BD, Zhang LS, Cho SJ, Bao S. Homeostatic plasticity drives tinnitus perception in an animal model. Proc Natl Acad Sci U SA. 2011 Sep;108(36):14974-9.

25. Friberg E, Jansson C, Mittendorfer-Rutz E, Rosenhall U, Alexanderson K. Sickness absence due to otoaudiological diagnoses and risk of disability pension: a nationwide Swedish prospective cohort study. PLoS One. 2012;7(1):e29966.

26. Lowe GD, Drummond MM, Forbes CD, Barbenel JC. The effects of age and cigarette-smoking on blood and plasma viscosity in men. Scott Med J. 1980 Jan;25(1):13-7.

27. Negley C, Katbamna B, CrumptonT, Lawson GD. Effects of cigarette smoking on distortion product otoacoustic emissions. J Am Acad Audiol. 2007 Sep;18(8):665-74.

28. Torre P 3rd, Dreisbach LE, Kopke R, Jackson R, Balough B. Risk factors for distortion product otoacoustic emissions in young men with normal hearing. J Am Acad Audiol. 2007 Oct;18(9):749-59.

29. Elgayar SA, Hussein OA, Abdel-Hafez AM, Thabet HS. Nicotine impact on the structure of adult male guinea pig auditory cortex. Exp Toxicol Pathol. 2016 Feb-Mar;68(2-3):167-79.

30. Liang K, Poytress BS, Chen Y, Leslie FM, Weinberger NM, Metherate R. Neonatal nicotine exposure impairs nicotinic enhancement of 
central auditory processing and auditory learning in adult rats. Eur J Neurosci. 2006 Aug;24(3):857-66.

31. Levine H, Berman T, Goldsmith R, Goen T, Spungen J, Novack L, et al. Exposure to tobacco smoke based on urinary cotinine levels among Israeli smoking and nonsmoking adults: a cross-sectional analysis of the first Israeli human biomonitoring study. BMC Public Health. 2013 Dec;13:1241.

32. CrinnionWJ.The CDC fourth national report on human exposure to environmental chemicals: what it tells us about our toxic burden and how it assist environmental medicine physicians. Altern Med Rev. 2010 Jul;15(2):101-9.

33. Matsumoto A, Matsumoto A, Ichiba M, Payton NM, Oishi H, Hara M. Simultaneous measurement of urinary total nicotine and cotinine as biomarkers of active and passive smoking among Japanese individuals. Environ Health Prev Med. 2013 May;18(3):244-50.

34. Jones-Burton C, Vessal G, Brown J, Dowling TC, Fink JC. Urinary cotinine as an objective measure of cigarette smoking in chronic kidney disease. Nephrol Dial Transplant. 2007 Jul;22(7):1950-4.

35. Wang Y, Yang M, Tian L, Huang Z, Chen F, Hu J, et al. Relationship between caregivers' smoking at home and urinary levels of cotinine in children. Int J Environ Res Public Health. 2014 Dec;11(12): 12499-513.
36. Lu L, Mackay DF, Newby DE, Pell JP. Association between salivary cotinine and cardiovascular biomarkers among nonsmokers and current smokers: cross-sectional study of 10,081 participants. Eur J Vasc Endovasc Surg. 2014 Dec;48(6):703-10.

37. Zierk J, Arzideh F, Haeckel R, Cario H, Fruhwald MC, Grob HJ, et al. Pediatric reference intervals for alkaline phosphatase. Clin Chem Lab Med. 2017 Jan;55(1):102-10.

38. Fischer DC, Mischek A, Wolf S, Rahn A, Salweski B, Kundt G, et al. Paediatric reference values for the C-terminal fragment of fibroblastgrowth factor-23, sclerostin, bone-specific alkaline phosphatase and isoform $5 \mathrm{~b}$ of tartrate-resistant acid phosphatase. Ann Clin Biochem. 2012 Nov;49(Pt 6):546-53.

39. Erlandsson SI, Holgers KM. The impact of perceived tinnitus severity on health-related quality of life with aspects of gender. Noise Health. 2001;3(10):39-51.

40. Nater UM, Abbruzzese E, Krebs M, Ehlert U. Sex differences in emotional and psychophysiological responses to musical stimuli. Int J Psychophysiol. 2006 Nov;62(2):300-8.

41. Vanneste S, Joos K, De Ridder D. Prefrontal cortex based sex differences in tinnitus perception: same tinnitus intensity, same tinnitus distress, different mood. PLoS One. 2012;7(2):e31182. 